\title{
Identification of a Redox Active Thioquinoxalinol Sulfate Compound Produced by an Anaerobic Methane-Oxidizing Microbial Consortium
}

\author{
Robert H. White, ${ }^{, \dagger}{ }^{\dagger}$ Kylie D. Allen, ${ }^{\dagger}$ and Gunter Wegener ${ }^{\sharp, \S}$ \\ ${ }^{\dagger}$ Department of Biochemistry, Virginia Polytechnic Institute and State University, Blacksburg, Virginia 24061, United States \\ ${ }^{\ddagger}$ MARUM, Center for Marine Environmental Sciences, 28359 Bremen, Germany \\ ${ }^{\S}$ Max Planck Institute for Marine Microbiology, University Bremen, 28359 Bremen, Germany
}

Supporting Information

ABSTRACT: The anaerobic oxidation of methane (AOM) mitigates the flux of methane from marine sediments into the water column. AOM is performed by anaerobic methanotrophic archaea (ANME) that reverse the methanogenesis pathway and partner bacteria that utilize the released reducing equivalents for sulfate reduction. Here, we investigated small-molecule extracts from sediment-free thermophilic enrichment cultures of ANME-1 and sulfate-reducing bacteria using ultraperformance liquid chromatography with high-resolution mass spectrometry. During the analysis, we discovered a novel thioquinoxalinol-containing redox molecule as a major component of the chemically derivatized small-molecule pool. This compound contains both a redox active quinoxaline heterocyclic ring and a thiol group. Additionally, the same core structure was identified that contains a sulfate ester on the hydroxyl group, which likely makes the molecule more water soluble. Hydrated versions of both structures were also observed as major compounds in the extracts. On the basis of reactions of model compounds such as quinoxalin-6-ol, the hydrated version appears to be formed from the addition of water to the dehydropyrazine

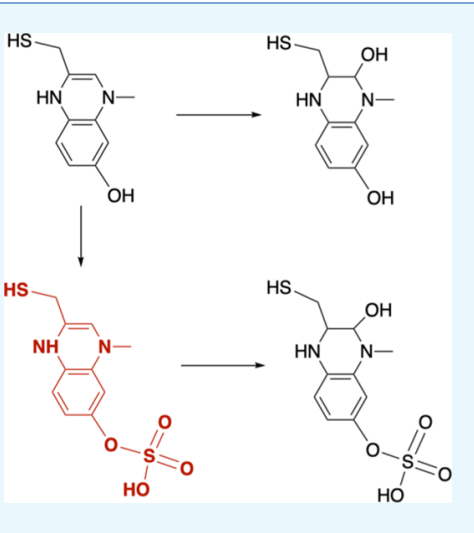
ring followed by an oxidation. These thioquinoxalinol compounds, which represent completely new structures in biochemistry, may be involved in electron transport processes within and/or between ANME-1 and sulfate-reducing bacteria, may serve protective roles by reacting with toxic compounds such as hydrogen sulfide, or may transport sulfate as a sulfate ester into the sulfate-reducing bacteria.

\section{INTRODUCTION}

Organisms across all domains of life produce an extensive suite of small molecules that play critical roles in life processes. For example, coenzymes such as pyridoxal phosphate and thiamine-pyrophosphate allow enzymatic reactions to occur that are not possible by proteins alone, while others including coenzyme $A$, coenzyme $M$, vitamin $B_{12}$, folates, and methanopterin, function as carrier molecules for metabolic intermediates. Furthermore, completely new coenzymes or modifications to currently known coenzymes continue to be discovered. $^{1-3}$ Other small biomolecules are involved in essential processes such as electron transfer carried out by flavin- and nicotinamide-containing cofactors, biochemical signaling such as homoserine lactones in quorum sensing, or metal-binding performed by siderophores.

The anaerobic oxidation of methane (AOM) with sulfate as the terminal electron acceptor is carried out by anaerobic methanotrophic archaea (ANME) existing in consortia with syntrophic bacteria in marine anaerobic environments. Given the key role of methane in the global carbon cycle as well as its concerning and increasing role as a greenhouse gas, a detailed biochemical understanding of methane sources and sinks is critical. ANME utilize a reverse methanogenic pathway to oxidize methane to $\mathrm{CO}_{2}$, thereby limiting the amount of methane that would otherwise be released into the atmosphere. ${ }^{4}$ Because of the lack of electron sinks, ANME shuttle reducing equivalents via an unconfirmed mechanism to sulfate-reducing bacteria (SRB), which use the electrons in their own energy metabolisms to reduce sulfate to sulfide. ${ }^{5-7}$ Sulfate-coupled AOM is performed by three different described clades of ANME that vary widely in terms of phylogeny, ecology, and physiology. ${ }^{7,8}$ The metagenome of ANME-1 from Black Sea mats was first published in 2010, ${ }^{9}$ followed by genomes of meso- and thermophilic ANME-1 archaea. ${ }^{10}$ In general, the presence of partner bacteria is essential for ANME activity, whereas at least Candidatus Desulfofervidus auxilii, the partner bacteria of thermophilic ANME-1, can be cultured independently in the presence of hydrogen and sulfate as the sole redox couple. ${ }^{11}$ Current evidence indicates that direct interspecies electron transfer occurs between ANME and SRB, as opposed to metabolic byproducts, such as hydrogen or formate, being produced by ANME and consumed by the partner bacteria. ${ }^{12-16}$ Electron transfer is likely mediated by small molecule and/or protein bound redox carriers, and the transfer may be facilitated by intercellular wiring consisting of pili-like connecting struc-

Received: October 16, 2019

Accepted: November 28, 2019

Published: December 16, 2019 
tures. ${ }^{13}$ The presence of multiheme cytochromes encoded in the genomes of ANME supports the involvement of these proteins in interspecies electron transfer, ${ }^{13,16}$ but this has not been confirmed and other electron carriers may also be involved.

Here, we studied the small-molecule molecular inventory of thermophilic AOM enrichments containing ANME-1 and Ca. D. auxilii partner bacteria. These microbial consortia have extremely low growth rates and yields, with doubling times of about 2 months. ${ }^{17}$ Thus, investigating their biochemistry is difficult because obtaining enough cellular material for many traditional biochemical analyses is currently out of reach. Therefore, here we take advantage of the sensitivity of mass spectrometry (MS) to analyze and characterize small molecules present in these consortia to reveal unique biochemical insights into AOM.

In our nontargeted analysis by ultraperformance liquid chromatography-high-resolution electrospray ionization-MS (UPLC-HR-ESI-MS), we identified and characterized a series of novel biomolecules. We propose chemical structures for the four related molecules containing a common thioquinoxalinol core structure (Figure 1). The thioquinox-

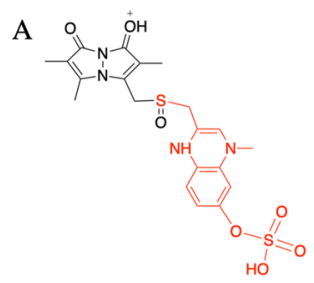

thioquinoxalinol sulfate derivative

Chemical Formula: $\mathrm{C}_{20} \mathrm{H}_{23} \mathrm{~N}_{4} \mathrm{O}_{7} \mathrm{~S}_{2}^{+}$
Exact Mass: measured $(\mathrm{M}+\mathrm{H})^{+}=495.101$

C
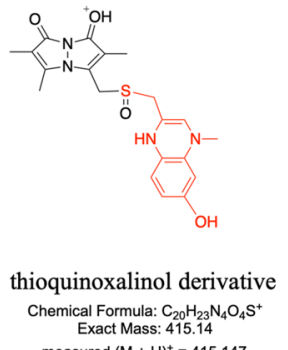
measured $(M+H)^{+}=415.147$

Figure 1. Proposed chemical structures of the bimane-sulfoxide derivatized thioquinoxalinol-containing molecules $(A-D)$ identified in this work. The core parts of the molecules obtained from the cells are in red, while the chemically derivatized portions are in black. The molecules are shown in their protonated forms as detected by ESIMS.

alinol structure has some similarities to the methanophenazine electron carrier first isolated in Methanosarcina mazei, ${ }^{18}$ as well as $\mathrm{FAD}, \mathrm{NAD}$, and menaquinone. However, unlike these established redox cofactors, the thioquinoxalinol molecules described here also have a thiol group, which could be involved in connecting thiol redox reactions with the reduction of the quinoxaline ring.

\section{RESULTS AND DISCUSSION}

Summary of the Identification of the Proposed Thioquinoxalinol-Containing Compounds. This work was specifically undertaken to explore AOM cultures for novel low molecular weight molecules that would reveal new biochemistry occurring within and/or between ANME-1 and their SRB partners. In our established workflow, water/ methanol-soluble cell extracts were prepared and the mass spectral data were analyzed for the presence of potentially unique molecules identified based on their measured exact masses and isotopic peak abundances. We then attempt to determine structures by comparing with similar known molecules and finally by the chemical synthesis of the molecule of interest or the synthesis of structural analogues.

Because we were specifically interested in thiol-containing compounds in this work, the cell extracts from ANME-1/SRB consortia were derivatized with bimane. This procedure results in thioethers and shields the compounds from oxidation when exposed to air. The resulting thioether from the bimane derivatization was subsequently oxidized to the sulfoxide and then analyzed by UPLC-HR-ESI-MS. Several major peaks were observed in the total ion chromatogram that appeared to be related compounds of unknown identity (Figure 2). The characteristics of the oxidized bimane derivatives were used to establish that all of the molecules contained a single thiol group. Based on the exact masses of the molecules, their fragmentation patterns, and the measured isotopic cluster intensities of the intact molecules and their fragments, we were able to propose likely structures for four related thioquinoxalinol-containing molecules. The basic structure contains a reduced thioquinoxalinol core with a thiol and a methylated nitrogen (Figure 1). The most abundant molecule observed, and likely the native biologically-active version, has a sulfateester (Figure 1A). Finally, hydrated versions of each of these were also observed as major peaks (Figure 1B,D). Each of the four compounds identified were observed as multiple peaks (Figure 2) with the retention times and masses indicated in Table 1, with each peak having the same masses and very similar fragmentation patterns, indicating the formation of different stable isomers. These compounds were not detected in growth media after the growth of these cells nor were they detected in the cells of methanogens or their spent media. In the next sections, we will elaborate on the structural characterization of these novel biomolecules.

Characteristics of the Bimane-Sulfoxide Form of Thioquinoxalinol Sulfate. The first thioquinoxalinol-containing molecule that we identified and characterized was the bimane-sulfoxide derivatized thioquinoxalinol sulfate (Figure 1A). From the measured mass of 495.101 and the fact that it contained two sulfurs, the elemental formula of the derivative was likely $\mathrm{C}_{20} \mathrm{H}_{23} \mathrm{~N}_{4} \mathrm{O}_{7} \mathrm{~S}_{2}$. The mass spectrum of the $10.86 \mathrm{~min}$ eluting peak is shown in Figure 3. Because the sample had been converted into a sulfoxide, this left the original thioquinoxalinol sulfate with an elemental formula of $\mathrm{C}_{10} \mathrm{H}_{12} \mathrm{~N}_{2} \mathrm{O}_{4} \mathrm{~S}_{2}$. The calculated mass for thioquinoxalinol sulfate measured from the $(\mathrm{M}+\mathrm{H})^{+}$from each peak observed in the order eluted in Figure 2 were 494.091, 494.091, 494.095, and 494.093. The isotopic intensities measured for the $(\mathrm{M}+\mathrm{H})^{+}$ion were $\mathrm{M}+1$ $=20 \%, \mathrm{M}+2=9.6 \%$, and $\mathrm{M}+3=1.8 \%$; from the $(\mathrm{M}+\mathrm{Na})^{+}$ ion were $M+1=20 \%, M+2=9.8 \%$, and $M+3=1.1 \%$; and from the $\mathrm{M}^{+}-\mathrm{SO}_{3}$ ion was were $\mathrm{M}+1=20 \%, \mathrm{M}+2=$ $5.36 \%$, and $\mathrm{M}^{+}+3=0.7 \%$. Taken together, these isotopic 


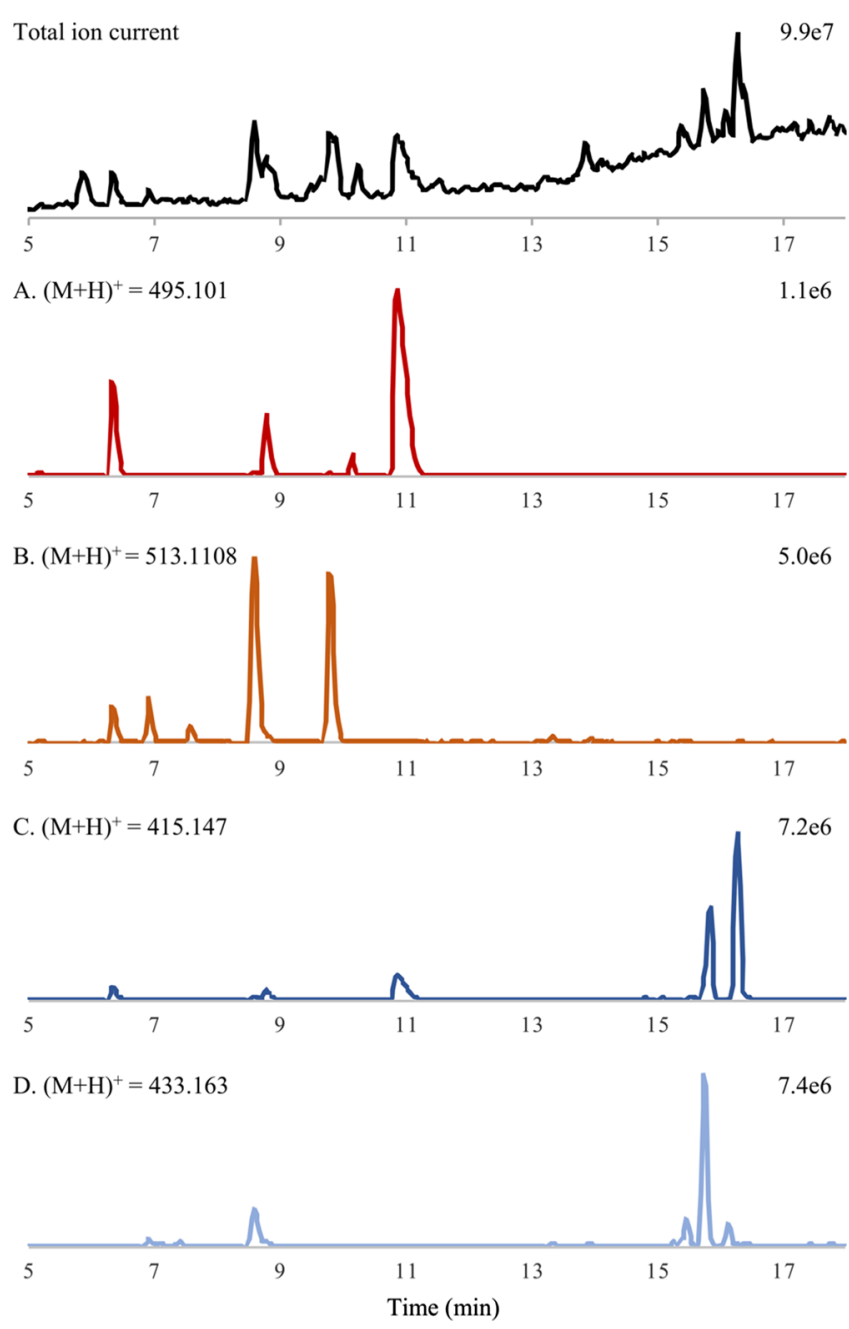

Figure 2. Extracted ion current LC-MS chromatograms for the bimane-sulfoxide derivatized thioquinoxalinol-containing compounds. The first three minor peaks in the 415.147 trace result from the fragmentation of the four compounds in the 495.101 trace. The data are summarized in Table 1. abundances confirm that the parent molecule contained two sulfur atoms and the $\mathrm{M}^{+}-\mathrm{SO}_{3}$ ion contains one sulfur. The peaks containing thioquinoxalinol sulfate also showed $\left(\mathrm{M}_{2}+\right.$ $\mathrm{H})^{+}=$989.190, $\left(\mathrm{M}_{2}+\mathrm{Na}\right)^{+}=1011.171,\left(\mathrm{M}_{3}+\mathrm{H}\right)^{+}=$ 1483.294, $\left(\mathrm{M}_{3}+\mathrm{Na}\right)^{+}=1505.270$, and $\left(\mathrm{M}_{3}+\mathrm{K}\right)^{+}=1521.242$, further confirming the mass of the parent molecule. An unexpected observation was that only some of the isomers formed both $\left(\mathrm{M}_{2}+\mathrm{H}\right)^{+}$at 989.190 and $\left(\mathrm{M}_{3}+\mathrm{H}\right)^{+}$ions (Table 1 ), which we currently do not have an explanation for.

The mass spectra of the molecules contained an intense 193.096 fragment ion (Figure 3A), arising from the bimanesulfoxide derivative, as the base peak. This 193.096 ion is observed along with 191.084 and 192.085 in the ESI fragmentation of thiol-containing compounds derivatized with bimane. The 193 ion occurs as a major ion in $~ 40 \%$ of molecules producing these three ions, but never as the base peak. This fragmentation ion would originate by an elimination of a reduced methylbimane with the formation of a sulfine (Figure 3B). The occurrence of such a fragment in other molecules has been previously observed. ${ }^{19}$ These data indicate that the carbon atom attached to the sulfoxide and quinoxalinol contained at least one hydrogen.

The mass spectrum of the bimane-sulfoxide derivative of thioquinoxalinol sulfate has a strong fragment ion at 415.147 (Figure 3A). This corresponds to a mass loss of 79.968 corresponding to the neutral loss of $\mathrm{SO}_{3}$ from the $(\mathrm{M}+\mathrm{H})^{+}$ ion, indicating a possible sulfate ester-containing molecule. Importantly, this fragment could not arise from the loss of $\mathrm{PO}_{3}$ from a phosphate monoester because it would have a mass loss of 78.958 from the $(\mathrm{M}+\mathrm{H})^{+}$. Alkyl monosulfate esters are known to fragment with the loss of $\mathrm{H}_{2} \mathrm{SO}_{4}{ }^{20}$ Negative mode ESI-MS analysis of a series of aromatic sulfate esters revealed a neutral loss of $\mathrm{SO}_{3}$, whereas nonaromatic sulfate esters do not fragment in this way. ${ }^{21}$ These observations indicated that the molecule of interest here contained an aromatic sulfate ester. The reduction in the intensity of $(\mathrm{M}+\mathrm{H})^{+}+2$ from the presence of ${ }^{34} \mathrm{~S}$ in the $(\mathrm{M}+\mathrm{H})^{+}$ion compared with the $(\mathrm{M}+$ $\mathrm{H})^{+}-\mathrm{SO}_{3}$ ion indicated the fragment contained one of the two sulfurs present in the molecule. The intensity of the +2 isotope ion from the measured 989.190 corresponding to $\left(\mathrm{M}_{2}\right.$

Table 1. LC-MS Data for the mBBr Sulfoxide Derivatives of Thioquinoxalinol and Thioquinoxalinol Sulfate and Their Hydrated Forms Identified from the AOM Consortia Described Here ${ }^{a}$

\begin{tabular}{|c|c|c|c|c|c|}
\hline \multicolumn{6}{|c|}{ A. thioquinoxalinol sulfate $(M+H)^{+}=495.101$} \\
\hline elution times $(\mathrm{min})$ & & 6.33 & $8.78^{b}$ & 10.15 & $10.87^{b}$ \\
\hline ratios of $495 / 415$ intensities & & 1.12 & 0.86 & 3.88 & 1.12 \\
\hline 495.101 peak intensities & & $2.4 \times 10^{5}$ & $1.5 \times 10^{5}$ & $3.6 \times 10^{4}$ & $5.0 \times 10^{5}$ \\
\hline \multicolumn{6}{|c|}{ B. hydrated thioquinoxalinol sulfate $(\mathrm{M}+\mathrm{H})^{+}=513.1108$} \\
\hline elution times $(\mathrm{min})$ & 6.33 & 6.92 & 7.57 & $8.59^{b}$ & $9.78^{b}$ \\
\hline ratios of 513/433 intensities & nd & 7.38 & 83 & 5.96 & 167 \\
\hline 513.1108 peak intensity & $8.9 \times 10^{5}$ & $1.2 \times 10^{6}$ & $3.1 \times 10^{5}$ & $5.1 \times 10^{6}$ & $4.6 \times 10^{6}$ \\
\hline \multicolumn{6}{|c|}{ C. thioquinoxalinol $(\mathrm{M}+\mathrm{H})^{+}=415.147$} \\
\hline elution times $(\mathrm{min})$ & 15.85 & 16.27 & & & \\
\hline 415.147 peak intensity & $4.0 \times 10^{6}$ & $7.2 \times 10^{6}$ & & & \\
\hline \multicolumn{6}{|c|}{ D. hydrated thioquinoxalinol $(\mathrm{M}+\mathrm{H})^{+}=433.163$} \\
\hline elution times $(\mathrm{min})$ & 15.46 & 15.75 & 16.12 & & \\
\hline 433.154 peak intensity & $1.2 \times 10^{6}$ & $7.4 \times 10^{6}$ & $7.8 \times 10^{5}$ & & \\
\hline
\end{tabular}

${ }^{a}$ Each ion intensity is for the $(\mathrm{M}+\mathrm{H})^{+}$ions. ${ }^{b}$ Compounds showing a $\left(\mathrm{M}_{2}+\mathrm{H}\right)^{+}$ion. Each $(\mathrm{M}+\mathrm{H})^{+}$and $\left(\mathrm{M}_{2}+\mathrm{H}\right)^{+}$ions all showed the $(\mathrm{M}+$ $\mathrm{Na})^{+}$ions at the expected masses. The 495 and $513{ }^{34} \mathrm{~S}$ isotopic ions all indicated the presence of two sulfur atoms and the 415 and $433{ }^{34} \mathrm{~S}$ isotopic ions both indicated the presence of one sulfur atom. The recorded 495/415 and 513/433 ion intensity ratios indicate the different efficiencies at which the sulfated molecules lose $\mathrm{SO}_{3}$. nd $=$ not determined due to interference by another peak. 

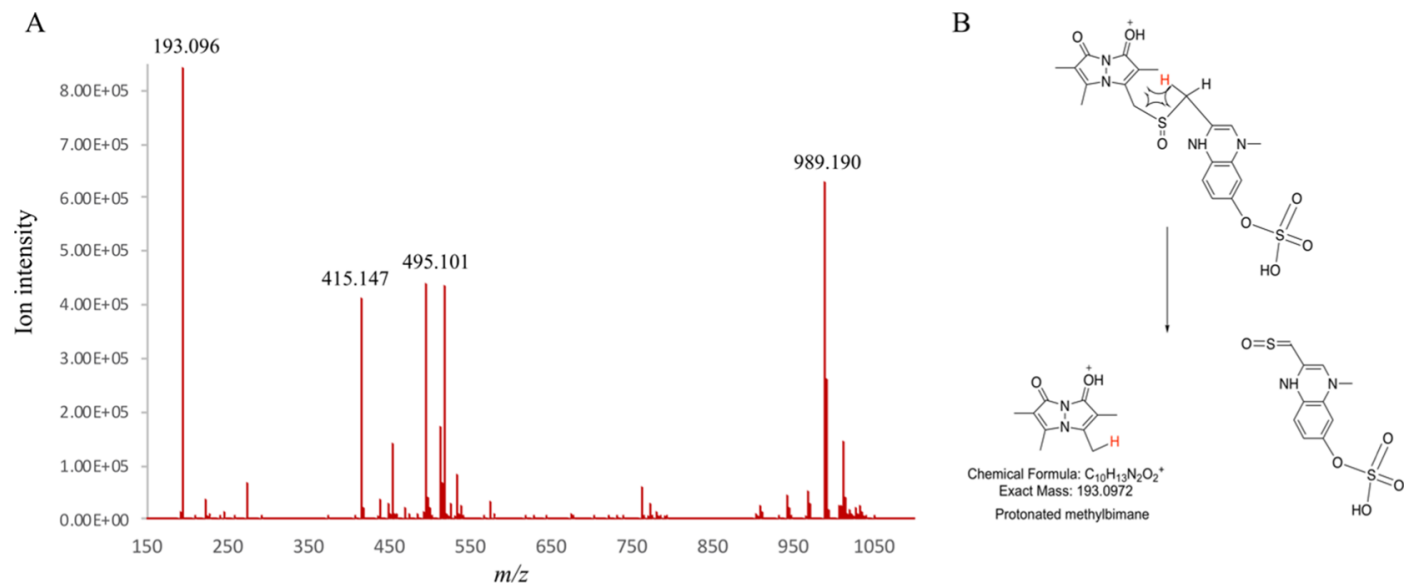

Figure 3. (A) Mass spectrum observed for the bimane sulfoxide derivative of thioquinoxalinol sulfate. The spectrum was that recorded at the elution time of $10.86 \mathrm{~min}$ in Figure 2. (B) Unexpected fragmentation of the bimane-sulfoxide derivative produced a substituted sulfine and protonated methylbimane, the latter of which is observed as the base peak in the mass spectrum $(\mathrm{m} / \mathrm{z}=193.096)$.

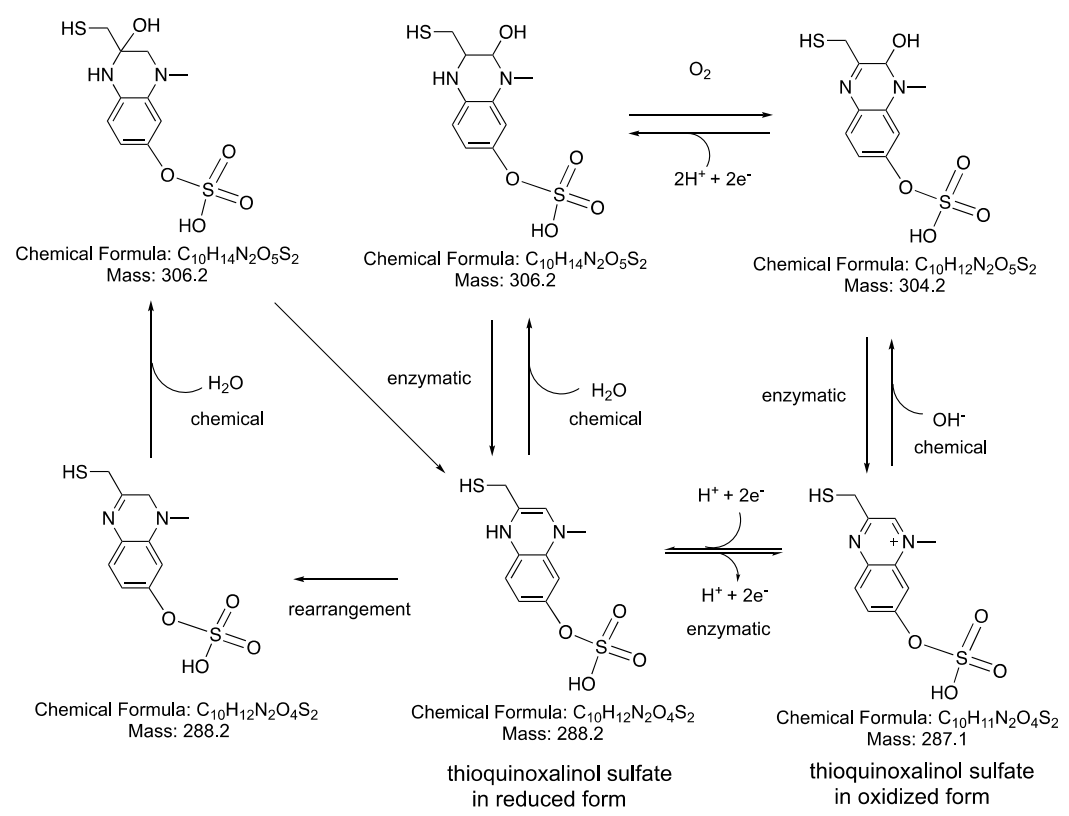

Figure 4. Possible routes for the enzymatic and chemical conversion of reduced thioquinalinol sulfate into the water addition products. The indicated reactions could occur either before or after the formation of the derivatives.

$+\mathrm{H})^{+}$again indicated the molecule contained two sulfur atoms.

Characteristics of the Bimane-Sulfoxide Form of Thioquinoxalinol. The bimane-sulfoxide form of the thioquinoxalinol without a sulfate-ester (Figure 1C) had a measured mass of 415.147. This would leave the mass of the core structure at 208.067 after the subtraction of the bimane and sulfoxide modifications and result in an elemental formula of $\mathrm{C}_{10} \mathrm{H}_{11} \mathrm{~N}_{2} \mathrm{OS}$. There are no compounds reported in SciFinder with this elemental formula that have the characteristics that we have described. Considering that the original structure must have one thiol group, one phenol, two nitrogens, and six sites of unsaturation, only a few biochemically relevant structures are possible; one is that proposed here (Figure 1C) and the others are positional isomers of this structure.

Taken together, the mass spectral data are consistent with the structures of the thioquinoxalinol molecules shown in Figure 1. Two major aspects of the structure remain ambiguous due to the fact that MS does not easily reveal positional isomers. The first is that the methyl group could be on the other nitrogen, and the second is that the hydroxyl (or sulfate) group could be on either the 7 th or 8 th position of the quinoxaline ring. The position of this hydroxyl group in our proposed structure is the same as that found in methanophenazine, which is a well-characterized redox molecule in methanogens. ${ }^{18}$ Analysis of a pure cell pellet of Ca. D. auxilii for the thioquinoxalinol compounds by thin-layer chromatography (TLC) and UV absorbance showed that they are below the limit of detection $(\sim 5 \mathrm{nmol} / \mathrm{g} \mathrm{dwt})$ in these samples. This indicates that the ANME in the consortia are producing the compounds, thus supporting their relation to methanophenazine in methanogens.

Evidence for the Proposed Structures Based on Comparisons to Synthetic Analogues. To further support the proposed structure of the molecule from the AOM consortia, we adapted literature procedures for the chemical synthesis of a thioquinoxalinol (Figure S1). The final 
derivatized synthetic product had a completely oxidized ring and thus was two mass units lower than the thioquinoxalinol isolated from the cells $(m / z 413.194$ vs 415.143$)$. The synthetic procedure yielded four different isomers (Figure S2). Mass spectral analysis of these synthetic products showed the characteristic protonated methylbimane fragment (Figure 3B). To provide further support for this base peak ion observed with the bimane-sulfoxide derivatives of the compounds studied here, the bimane-sulfoxide derivative of mercaptoethylpyrazine was prepared in the same manner as the samples from the cells and purified by preparative TLC. This molecule also produced the same $m / z 193.0972$ fragment, showing that this related model thiol compound also fragments to produce a protonated methylbimane.

We next wanted to investigate the potential redox properties of these newly discovered biomolecules. Because the above synthetic procedure yielded low amounts of the final products, we instead studied the properties of the core quinoxaline without the sulfate and thiol functionalities. Thus, quinoxaline6-ol was methylated with methyl iodide, and the products were assayed by TLC to reveal two colored spots which were purified by preparative TLC. LC-MS analysis revealed that the top spot was the starting material and the lower spot was the methylated product. The bottom spot contained two compounds both with a $\mathrm{M}^{+}=161.1$, resulting from having one methyl on the nitrogen on the same side as the hydroxyl group (7-hydroxy-1-methylquinoxalin-1-ium, Figure S3) and the other having the methyl group on the opposite side from the hydroxy group (7-hydroxy-1-methylquinoxalin-4-ium). This mixture was readily reduced with dithionite, and the UV-vis spectrum recovered upon exposure to air, thus demonstrating the redox active characteristics of the model compound.

Characterization of the Hydrated Forms of Thioquinoxalinol Derivatives. In addition to thioquinoxalinol sulfate, we also observed a series of peaks with the mass of bimane-sulfoxide thioquinoxalinol sulfate but corresponding to the addition of water. These molecules had a mass of 513.116 which is 18.0106 higher than thioquinoxalinol sulfate (Figure 1). In the UPLC-HR-ESI-MS measurements, five peaks with this mass were observed (Figure 2 and Table 1). Possible routes for the formation of these compounds from thioquinoxalinol sulfate are shown in Figure 4.

The reason for this addition of water is not clear, but the fact that five peaks were observed indicates that some of the formation represents side reactions not related to the true function of this molecule because the multiple peaks are a result of racemic products. To test this with our model compounds, the 7-hydroxy-1-methylquinoxalin-4-ium and 7hydroxy-1-methylquinoxalin-1-ium mixture was exposed to sodium hydroxide in the presence of air, which indeed generated a hydroxylated form (Figure S4A). Many examples exist where hydroxide is known to add to the electrophilic sites of oxidized coenzymes. A prime example of this is the addition of water to $\mathrm{NAD}^{+}{ }^{22}$ Additionally, diquaternary salts of pyrazines are known to undergo the addition of alcohols with no change in the redox state, ${ }^{23}$ and quinoxalines undergo redox reactions at a carbon electrode to produce water addition products as the result of the formation of a 1,4didehydro product. $^{25}$ It has also been reported that 1,4dihydroquinoxaline can undergo the addition of water during electrochemical studies but the mechanism is not clear. A similar mechanism has been reported for lumazine. ${ }^{24}$
Another route for the formation of the water adduct could start with the addition of hydrogen peroxide to the 7-hydroxy1-methylquinoxalin-4-ium and 7-hydroxy-1-methylquinoxalin1-ium mixture. Thus, we treated 7-hydroxy-1-methylquinoxalin-1-ium mixture with hydrogen peroxide and tris-(2carboxyethyl)phosphine hydrochloride (TCEP) as was done during the isolation of the cell extract sample. The product obtained was 7-hydroxy-1-methyl-3,4-dihydroquinoxalin$2(1 \mathrm{H})$-one (Figure S4B). We propose that a peroxide anion adds to the C-2 carbon of 7-hydroxy-1-methylquinoxalin-1-ium and the generated hydroperoxide is reduced to an alcohol with the TCEP, a well-known reaction. ${ }^{25}$ The resulting enamine then undergoes a rearrangement to form the enol alcohol that undergoes a rearrangement to form the stable product, 7hydroxy-1-methyl-3,4-dihydroquinoxalin-2(1H)-one. This compound cannot be oxidized back to 7-hydroxy-1-methylquinoxalin-1-ium.

Reason for the Occurrence of Several Different Isomers. As indicated in Table 1, each of the bimanesulfoxide containing derivatives showed multiple chromatographic peaks. The presence of these multiple peaks can be explained as a result of different stereochemistries at the chiral atoms (circled in Figure S5). Because the sulfoxides were generated by chemical oxidation, they will be composed of two possible stereoisomers. Furthermore, depending on the route for the water addition, additional chiral centers will be generated. These chiral centers will lead to diastereomers which will generate multiple LC peaks for both thioquinoxalinol, thioquinoxalinol sulfate, and their hydrated forms.

Analysis of Thioquinoxalinol Bimane Thioethers. We also analyzed cell extracts that were derivatized with bimane but not oxidized to the sulfoxide. Scanning of the data for the expected different forms of the bimane derivatives indicated the presence of three of the thioquinoxalinol-containing molecules (Figure S6). The most intense form detected was the hydrated form with no sulfate ester with a measured mass of 415.147. The next most abundant peak was the completely oxidized form of the intact compound with a measured mass of 477.0897. The least intense peak was the intact cofactor but with quinoxaline ring reduced by $\left(2 \mathrm{H}^{+}+2 \mathrm{e}^{-}\right)$with a measured mass of 479.1054 . The elution times of the molecules matched their expected polarities.

Abilities of Quinoxalines, Pyrazines, and Phenazines To Undergo Redox Reactions. Although the literature lacks data on the reduction potentials of any thioquinoxalinol molecules, data have been reported for comparable redox active compounds. The closest analogues are ubiquinone, phenazine methosulfate, and pyocyanin with established reduction potentials of $+113,^{26}+80,{ }^{27}$ and $-34 \mathrm{mV},{ }^{28}$ respectively. These data indicate that thioquinoxalinol is likely to have a mid-range reduction potential. The effect of the addition of the sulfate ester is hard to predict, and the redox properties could be substantially different if the molecule is bound to a protein.

Analysis by UV-vis spectrophotometry revealed that $\mathrm{N}$ methylated quinoxaline-6-ol is readily reduced by dithionite, whereas quinoxaline-6-ol and thioethylpyrazine cannot be reduced with dithionite. It thus appears that the introduction of the methyl group on the nitrogen to generate a quaternary nitrogen is required for the compound to be reduced by dithionite $(-660 \mathrm{mV})$. Quaternary salts of pyrazine ${ }^{29}$ and quinoxalines $^{30}$ can be $\mathrm{N}$-methylated with methyl iodide. The diquaternary salts can only be prepared using oxonium salts, 


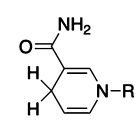

NADH

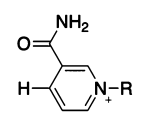

NAD

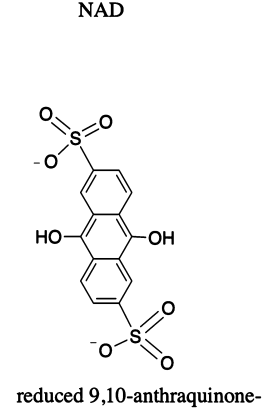
2,6-disulfonate

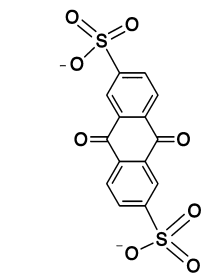

oxidized 9,10-anthraquinone2,6-disulfonate

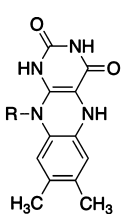

reduced flavin

o

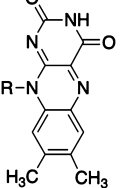

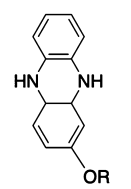

reduced methanophenzane ( $\mathrm{R}=$ isoprenoid)

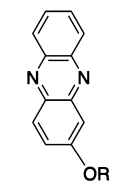

oxidized flavin oxidized methanophenzane

( $R=$ isoprenoid)

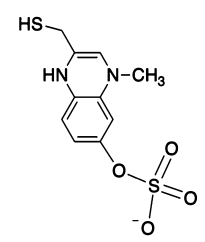

reduced thioquinoxalinol sulfate

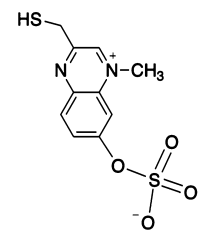

oxidized thioquinoxalinol sulfate

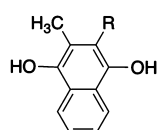

reduced menaquinone

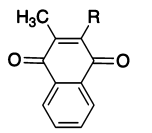

oxidized menaquinone

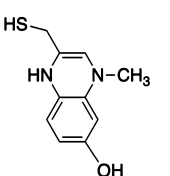

reduced thioquinoxalinol
HS

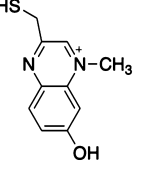

OR

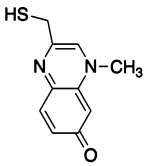

oxidized thioquinoxalinol(one)

Figure 5. Similarities in the chemical structures among the known redox coenzymes and thioquinoxalinol and its sulfate ester.

which are stronger alkylating reagents. ${ }^{23}$ These resulting methylated compounds can undergo redox reactions, ${ }^{31,32}$ thus supporting the role of the thioquinoxalinol molecule discovered here as a redox active compound.

5-Methylphenazonium methyl sulfate (phenazine methosulfate) is a well-established electron carrier that can replace flavins in flavoproteins. ${ }^{33}$ It undergoes reduction with NADH as well as other reducing agents. Phenazine itself can be readily reduced to 5,10-dihydrophenazine which is unstable in air, ${ }^{34}$ and such reduced phenazines readily reduce ferric (hydr)oxides, which may play an important role in the iron uptake. ${ }^{35}$ Pyrazine and quinoxaline heterocycles have established redox characteristics, where they can be reduced in aqueous solutions both electrochemically ${ }^{36}$ and with titanium(III) chloride ${ }^{37}$ and can undergo two one-electron transfers. ${ }^{38}$ Pyrazine is completely reduced to piperazine by nickel-aluminum alloy $^{39}$ and quinoxaline can also be reduced to $1,4-$ dihydroquinoxaline ${ }^{40-42}$ which appears to be stable in air. This is analogous to NADH which is also stable in air in the reduced form. Electrochemical reduction produces 1,4dihydropyrazines, which are unstable to isolate and isomerize into 1,2- or 1,6-dihydropyrazines. ${ }^{41}$ Hydroxyquinolines can participate in two-electron reactions to produce aminophenolquinoneimine tautomers, ${ }^{43}$ and dihydroquinoxalines disproportionate to the oxidized and reduced compounds. ${ }^{44}$ Thus, the reduction of the quinoxaline ring to 1,4-dihydroquinoxaline is the form of the thioquinoxalinol sulfate isolated in this study.

Function of Thioquinoxalinol and Its Sulfate Ester. Although it is not possible to report the actual concentrations of these thioquinoxalinol compounds based on the current data, the derivatized versions are the major peaks in the total ion chromatogram (Figure 2) and thus these compounds are likely major compounds with important functions in these consortia. If their ion intensities are compared to the intensity of coenzyme $F_{430}$ in the same sample and it is assumed that the amount of $\mathrm{F}_{430}$ is the same as reported for methanogens, ${ }^{45}$ then the combined amounts of the thioquinoxalinol-containing molecules was $\sim 36 \mathrm{mM}$ in the original cell pellet. Because the sensitivity of $\mathrm{F}_{430}$ detection by MS is very high due to the $\mathrm{Ni}(\mathrm{III})$ imparting a single positive charge, it is possible that the concentrations of the compounds reported here could be even higher.

Thioquinoxalinol and thioquinoxalinol sulfate have all of the hallmarks of redox carrier coenzymes (Figure 5). The core structure has chemical features comparable to methanophenzine, a membrane-bound electron carrier in methanogens. ${ }^{18}$ In the nonsulfated form, thioquinoxalinol could also undergo reactions analogous to quinones. The presence of the sulfate ester will make the thioquinoxalinol more water soluble and the thiol may allow it to transfer electrons thorough thiol groups. Because one of the nitrogens has an attached methyl group, then it has the appearance of a pyridine nucleotide with the C-4 being replaced with a nitrogen (Figure 5). The most intriguing component of the thioquinoxalinol molecules is the presence of both a redox active quinoxaline ring and thiol group. All of the known redox active coenzymes have only one redox center. 


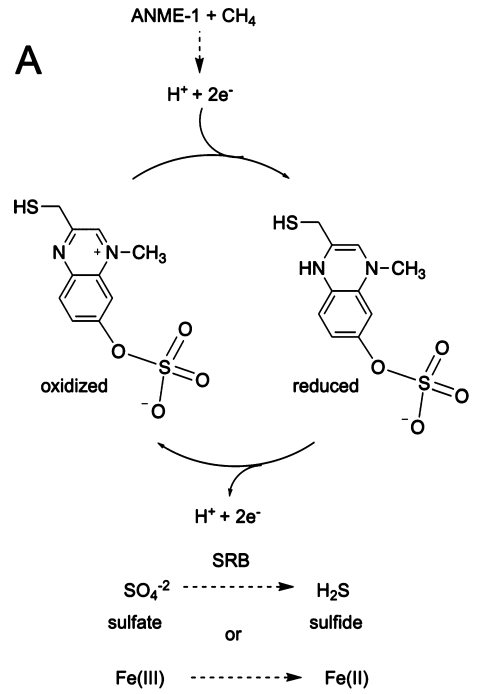

B

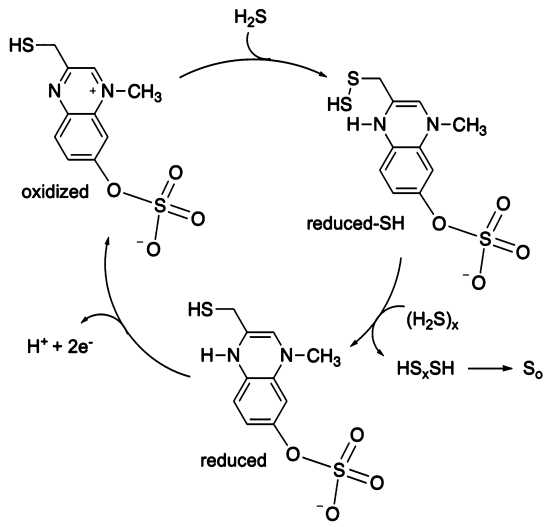

Figure 6. Possible functions of thioquinoxalinol sulfate. (A) Proposed electron transfer between ANME-1 and SRB assisted by the thioquinoxaline redox carrier. (B) Possible involvement of the thioquinoxaline redox carrier in the oxidation of $\mathrm{H}_{2} \mathrm{~S}$ to polysulfides and $\mathrm{S}_{8}$. This process would be involved in the removal toxic hydrogen sulfide.

An intriguing possible function of the thioquinoxalinol molecule(s) in the AOM consortia is to mediate electron transfer between ANME and SRB (Figure 6A). As discussed above, thioquinoxalinol likely has a reduction potential between about -50 to $+100 \mathrm{mV}$ based on established properties of structurally similar redox molecules. This indicates that it could not serve as a direct electron donor for the SRB partner in AOM consortia because electrons with potentials of around $-250 \mathrm{mV}$ are required. ${ }^{46,47}$ Interestingly, our proposed structure is very similar to the artificial electron acceptor, 9,10-anthraquinone-2,6-disulfonate (AQDS, Figure 5 ). AQDS has a sulfonic acid in place of a sulfate ester and an anthraquinone ring in place of the quinoxaline ring. This artificial molecule can be used as an electron acceptor in AOM consortia where it decouples methane oxidation from sulfate reduction, indicating that ANME can release electrons to extracellular electron acceptors to carry out methane oxidation. ${ }^{14}$ Additionally, Lovley reported in 2002 that some methanogens can use AQDS as an electron shuttle for Fe(III) reduction. ${ }^{48}$ This has been confirmed by recent work with Methanosarcina acetivorans. ${ }^{49}$ Because the expected reduction potential of thioquinoxalinol is within the range of ferrihydrite, $^{50}$ it is possible that these molecules discovered here are involved in $\mathrm{Fe}$ (III) reduction (Figure 6A).

In the potential roles described above, the thiol group has no clear functional requirement. A possible alternative or additional function is shown in Figure $6 \mathrm{~B}$, where the thioquinoxalinol is involved in sulfur metabolism. Here, hydrogen sulfide reduces the quinoxaline ring and the resulting sulfane sulfur reacts with the thiol to form a persulfide $(-\mathrm{SSH})$ which leads to the formation of $S_{8}$. The production of $S_{8}$ mediated by thioquinoxalinol sulfate would reduce the amount of toxic sulfide. This type of biochemistry is comparable to the established role of flavoproteins in the reduction of disulfides. $^{51}$ Once reduced, the thioquinoxalinol compound could be involved in oxygen detoxification. Finally, the identification of sulfated and nonsulfated thioquinoxalinol implicates a possible role as a sulfate carrier for delivering sulfate to the SRB.
Final Comments. One must consider that thioquinoxalinol represents a primitive coenzyme that originally had several redox functions. Once we have a proven route to its isomerically pure chemical synthesis, we will explore these functions.

\section{EXPERIMENTAL SECTION}

Chemicals. Quinoxalin-6-ol, 4-(benzyloxy)benzene-1,2-diamine hydrochloride, and mercaptoethylpyrazine was supplied by Enamine, Monmouth Jct., NJ. Methyl iodide, monobromobimane $(\mathrm{mBBr})$ and $\alpha$-thioglycerol was obtained from Sigma-Aldrich, St Louis MO. 4-Phenylbut-3-en-2-one and dichlorotris(triphenylphosphino)ruthenium(II) were obtained from Ambeed, Arlington Heights, IL. S-Benzylthioglycerol was prepared by the reaction of $\alpha$-thioglycerol with chlorotoluene. The final product was purified by chromatography on a $\mathrm{SiO}_{2}$ column by elution with a mixture of methylene chloride and methyl acetate mixtures. The details of the chemical synthesis of the thioquinoxalinol core structure with an oxidized quinoxaline is described in the Supporting Information and shown in Figure S1. This synthesis produced the expected four isomers that are shown in Figure S2.

Origin and Cultivation of AOM-Performing Consortia. The thermophilic AOM consortia were cultured from hydrothermal vent sediments collected from mat-covered sites in the Guaymas Basin, Gulf of California, Mexico $\left(27.7438^{\circ} \mathrm{N}\right.$, $\left.111.409133^{\circ} \mathrm{W}\right)^{17}$ during RV Atlantis cruise AT15-56 in November/December 2009 (Alvin Dive 4570). At the Max Planck Institute for Marine Microbiology, Bremen, Germany, sediments were diluted with sulfate reducer medium prepared after Widdel and Bak. ${ }^{52,53}$ We provided methane $(0.225 \mathrm{MPa}$ $\left.\mathrm{CH}_{4}(\mathrm{~g})\right)$ and sulfate $\left(28 \mathrm{mM} \mathrm{SO}_{4}{ }^{-2}(\mathrm{aq})\right)$ as sole electron donor and acceptor, respectively, and carbon dioxide ( 0.025 $\mathrm{MPa} \mathrm{CO}_{2} ; 30 \mathrm{mM}$ dissolved inorganic carbon) as a carbon source. Samples were incubated at $50{ }^{\circ} \mathrm{C}$ and culture media were exchanged when sulfide concentrations exceeded $\sim 12$ $\mathrm{mM}$ and samples were regularly diluted $(1: 2 ; 1: 4)$, reaching sediment-free states after 3 years. To determine the molecular inventory of the cultures, $450 \mathrm{~mL}$ of culture was harvested at about $10 \mathrm{mM}$ sulfide content. Under anoxic conditions, cells 
were centrifuged and cell pellets were immediately frozen. Additionally, fresh and highly sulfidic incubation media were analyzed. ANME-1 containing cell consortia were separated from the media and cells, and the spent media were sent from Germany to the US at $-80{ }^{\circ} \mathrm{C}$.

Extraction of Cell Pellets. The extraction procedure is diagramed in Figure S7 and described here. The black pellet (0.283 g wet weight) containing the ANME-1/SRB cells was placed in a sealed tube with $3 \mathrm{~mL}$ of $60 \%$ methanol in water, and the sample was heated with shaking for $5 \mathrm{~min}$ at $100{ }^{\circ} \mathrm{C}$. This heating ensured that all proteins were denatured so as to release all bound cofactors. After cooling, the samples were centrifuged and the pellets were re-extracted in the same manner a second time. The resulting red extracts were combined and evaporated to $1 \mathrm{~mL}$ with a stream of nitrogen and large molecules were removed from the sample using an Amicon Ultra- $0.5 \mathrm{~mL} 3 \mathrm{~K}$ centrifugal filter. (The red color of the extracts likely comes from the resazurin added to the growth media.) After evaporation of the samples to a volume of $\sim 70 \mu \mathrm{L}$, a portion of this sample was assayed directly by UPLC-HR-ESI-MS.

Analysis of Cell Content in the Black Pellet Based on the Amount of Protein Extracted. A $51.4 \mathrm{mg}$ portion of above black wet pellet was dried to produce $10.3 \mathrm{mg}$ of a dark gray solid, indicating that the pellet was $80 \%$ water. Extraction of this solid with $100 \mu \mathrm{L}$ of $0.5 \mathrm{M} \mathrm{NaOH}$ for $5 \mathrm{~min}$ at $100{ }^{\circ} \mathrm{C}$ released $0.5 \mathrm{mg}$ of protein (Figure S7). Based on established data showing that $55 \%$ of the dried weight of Escherichia coli is protein, one can calculate that only $\sim 1.1 \mathrm{mg}$ of the dried pellet was from cells. Considering that $0.283 \mathrm{~g}$ wet weight of cell pellet was used, then the data reported here were derived from only $6 \mathrm{mg}$ dry weight of cells. The bulk of the sample has been identified using Raman spectroscopy as crystals of magnesium phosphate $\mathrm{Mg}_{3}\left(\mathrm{PO}_{4}\right)_{2} \cdot 8 \mathrm{H}_{2} \mathrm{O}$ (bobierrite), $\mathrm{Fe}_{3}\left(\mathrm{PO}_{4}\right)_{2} \cdot 8 \mathrm{H}_{2} \mathrm{O}$ (vivianite), $\mathrm{FeS}_{2}$, and amorphous carbon.

Formation of Derivatives. The remaining cell extract was treated with $\mathrm{mBBr}$ that converted all thiols into fluorescent thioethers. The methods for the modification of the thiol groups were developed following those described by Fahey, ${ }^{54}$ which have also been modified for the analysis of sulfite and thiosulfate in sea water ${ }^{55}$ and for the identification of 3mercaptopropionic acid in methanogens. ${ }^{56}$ To the concentrated sample, $20 \mu \mathrm{L}$ of $50 \mathrm{mM}$ TCEP (adjusted to $\mathrm{pH} 7$ with $\mathrm{NaOH}$ ), $20 \mu \mathrm{L}$ of $0.1 \mathrm{M}$ borate buffer ( $\mathrm{pH} 9.3$ ), and $20 \mu \mathrm{L}$ of $0.1 \mathrm{M}$ solution $\mathrm{mBBr}$ in acetonitrile were added. The TCEP was added to reduce any disulfides formed during aerobic sample preparation. Samples were then assayed for the presence of thiol-containing compounds using UPLC-HRESI-MS as preciously described. ${ }^{56}$

After completion of the two assays above, the remaining sample was reacted with a molybdate-containing catalyst in the presence of peroxide. ${ }^{57}$ This reaction specifically converts molecules containing thioethers into sulfoxides. This transformation occurred in those molecules where the thiol groups had been converted into bimane derivatives. In this procedure, $3 \mu \mathrm{L}$ of $0.1 \mathrm{M} \mathrm{H}_{2} \mathrm{O}_{2}$ and $3 \mu \mathrm{L}$ of $0.2 \mu \mathrm{M}$ of the Mo reagent were added to $40-70 \mu \mathrm{L}$ of the bimane derivatized sample with mixing. After $20 \mathrm{~min}$ at room temperature, $3 \mu \mathrm{L}$ of a 0.1 $\mathrm{M}$ solution of thiosulfate was added and after 2 or more hours, the sample was assayed by UPLC-HR-ESI-MS. The sample was then assayed a third time to confirm the presence of sulfoxides. This was done to further confirm that the original molecule contained a thiol group.
Chemical Synthesis and Characterization of Model Compounds. Quinoxalin-6-ol was monomethylated at one of the two nitrogens with methyl iodide (Figure S3). Thus, $5 \mathrm{mg}$ of quinoxalin-6-ol was mixed $100 \mu \mathrm{L}$ of methyl iodide, $100 \mu \mathrm{L}$ of methylene chloride, and $100 \mu \mathrm{L}$ of acetonitrile and heated at $60{ }^{\circ} \mathrm{C}$ for $1 \mathrm{~h} .^{58}$ The resulting red sample was then evaporated, and the residue was dissolved in water and purified by preparative TLC using the solvent consisting of 5\% formic acid in acetonitrile. In this solvent system, two major colored spots with UV absorbance were observed in about equal amounts. The top TLC spot was yellow and the lower spot was red. These spots were scraped from the TLC plate eluted with the TLC solvent and their MS and absorbance spectra obtained. The top yellow band $\left(R_{\mathrm{f}}=0.61\right)$ was same as quinoxalin-6-ol and had the same UV absorbance spectra $\left(248 \mathrm{~nm} \lambda_{\max } 232\right.$, and $350 \mathrm{~nm}$ in methanol) as the starting material. LC-MS showed a single peak with $(\mathrm{M}+\mathrm{H})^{+}=147.1$. The bottom red band had a $R_{\mathrm{f}}=0.23$ and had a $\lambda_{\max }=275 \mathrm{~nm}$ in water. A solution of the bottom band in anaerobic $50 \mathrm{mM}$ TES buffer with $10 \mathrm{mM} \mathrm{MgCl}_{2}$ at $\mathrm{pH} 7.3$ had absorbance peaks of 275 , 335 , and $469 \mathrm{~nm}$ with the relative intensities of 1.0, 0.10, and 0.32 , respectively. As expected, the TLC-purified lower band showed two LC-MS peaks both with a $\mathrm{M}^{+}=161.1$ resulting from having one methyl on the nitrogen on the same side as the hydroxyl group and the other having the methyl group on the opposite side from the hydroxyl group. We did not try to establish which TLC spot was which positional isomer. LCMS spectral data for these compounds were recorded on an $A B$ Sciex Quadrupole MS interfaced with an Agilent 1200 series LC as previously reported. ${ }^{56}$

The original quinoxalin-6-ol sample had an absorbance spectrum that was unchanged in the presence of dithionite, indicating the compound could not be reduced by dithionite. On the other hand, the lower purified TLC spot containing the two N-methylated quinoxaline-6-ol isomers was reduced with dithionite in water. This was confirmed by the loss of the red color. Shaking the sample in air for a few seconds completely restored the original spectrum and the red color, indicating that the methylated quinoxaline ring was redox active. In contract, a solution of mercaptoethylpyrazine exposed to dithionite showed no reduction.

A sample of the monomethylated quinoxalin-6-ol was mixed with $25 \mu \mathrm{L}$ of $1 \mathrm{M} \mathrm{NaOH}$, and the sample was heated at $60^{\circ} \mathrm{C}$ for $6 \mathrm{~h}$. After the addition of $2 \mu \mathrm{L}$ of HOAc, the resulting sample was purified by preparative TLC using $5 \% \mathrm{HCOOH}$ in $\mathrm{CH}_{3} \mathrm{CN}$. The product with $\mathrm{R}_{\mathrm{f}}=0.63$ was eluted from the plate and assayed by UPLC-HR-ESI-MS to show two peaks with $\mathrm{M}^{+}=177.067$ corresponding to $\mathrm{C}_{9} \mathrm{H}_{9} \mathrm{~N}_{2} \mathrm{O}_{2}$. These correspond to the structures shown in Figure $\mathrm{S} 4$.

UPLC-HR-ESI-MS Analysis of Samples. Samples were analyzed on a Waters SYNAPT G2-S high-definition mass spectrometer connected to a Waters Acquity UPLC I-class system with an Acquity UPLC BEH C18 (Waters, $2.1 \mathrm{~mm} \times$ $75 \mathrm{~mm}, 1.7 \mu \mathrm{m}$ particle size) column. Solvent A was $0.1 \%$ formic acid in water and solvent B was $100 \%$ acetonitrile. The flow rate was $0.2 \mathrm{~mL} / \mathrm{min}$ and gradient elution was employed in the following manner $(t(\min ), \% \mathrm{~B}):(0.01,5),(6,15),(21$, $35),(23,65)$. For each analysis, $10 \mu \mathrm{L}$ of the sample was injected. The mass spectral data were collected in highresolution MSe continuum mode. A lock spray scan (function 3) was collected every $20 \mathrm{~s}$ for calibration, and the lock spray analyte used was leucine-enkephalin. Parameters were $2.8 \mathrm{kV}$ capillary voltage, $125{ }^{\circ} \mathrm{C}$ source temperature, $350{ }^{\circ} \mathrm{C}$ 
desolvation temperature, $35 \mathrm{~V}$ sampling cone, $50 \mathrm{~L} / \mathrm{h}$ cone gas flow, $500 \mathrm{~L} / \mathrm{h}$ desolvation gas flow, and $6 \mathrm{~L} / \mathrm{h}$ nebulizer gas flow. The collision energies for the low-energy scans (function 1) were 4 and $2 \mathrm{~V}$ in the trap region and the transfer region, respectively. Collision energies for the high-energy scans (function 2) were ramped from 25 to $45 \mathrm{~V}$ in the trap region and $2 \mathrm{~V}$ in the transfer region. Data were analyzed using MassLynx program (Waters). The extracted ion chromatograms for the molecules in Figure 1 are shown in Figure 2.

Safety Statement. No unexpected or unusually high safety hazards were encountered.

\section{ASSOCIATED CONTENT}

\section{(S) Supporting Information}

The Supporting Information is available free of charge at https://pubs.acs.org/doi/10.1021/acsomega.9b03450.

Chemical synthesis of thioquinoxalinol; structures of the four possible positional isomers produced in the first two chemical steps in the synthesis of the model thioquinoxalinol cofactor; chemical synthesis used to generate two mono $\mathrm{N}$-methylated isomers of quinoxaline-6-ol; model reactions to explain the formation of the hydrated forms from one of the isomers of 7-hydroxy-1-methylquinoxaline-1-ium; proposed explanation for the generation of multiple isomers for the bimanesulfoxide thioquinoxalinol sulfate and its water addition products; proposed structures of the bimane derivatives lacking the sulfoxide; and outline of procedure used to measure protein in the black pellet (PDF)

\section{AUTHOR INFORMATION}

\section{Corresponding Author}

*E-mail: rhwhite@vt.edu. Phone: (540) 231-6605.

\section{ORCID $\odot$}

Robert H. White: 0000-0002-9105-7445

Notes

The authors declare no competing financial interest.

\section{ACKNOWLEDGMENTS}

This work was supported by the National Science Foundation Grant MCB1120346 to R.H.W. The authors thank Dr. W. Keith Ray for performing the UPLC-HR-ESI-MS measurements. The MS resources are maintained by the Virginia Tech Mass Spectrometry Incubator, a facility operated in part through funding by the Fralin Life Science Institute at Virginia Tech and by the Agricultural Experiment Station Hatch Program (CRIS project number: VA-135981).

\section{ABBREVIATIONS}

ANME, anaerobic methanotrophs; AOM, anaerobic oxidation of methane; SRB, sulfate-reducing bacteria; UPLC-HR-ESIMS, ultraperformance-liquid-chromatography-high-resolution electrospray ionization-mass spectrometry; TCEP, tris-(2carboxyethyl)phosphine hydrochloride; $\mathrm{B}$, monobromobimane

\section{REFERENCES}

(1) Piano, V.; Palfey, B. A.; Mattevi, A. Flavins as Covalent Catalysts: New Mechanisms Emerge. Trends Biochem. Sci. 2017, 42, 457-469.

(2) Fahey, R. C. Novel thiols of prokaryotes. Annu. Rev. Microbiol. 2001, 55, 333-356.

(3) Allen, K. D.; Wegener, G.; White, R. H. Discovery of multiple modified $\mathrm{F}_{430}$ coenzymes in methanogens and anaerobic methano- trophic archaea suggests possible new roles for $\mathrm{F}_{430}$ in nature. Appl. Environ. Microbiol. 2014, 80, 6403-6412.

(4) Bhattarai, S.; Cassarini, C.; Lens, P. N. L. Physiology and Distribution of Archaeal Methanotrophs That Couple Anaerobic Oxidation of Methane with Sulfate Reduction. Microbiol. Mol. Biol. Rev. 2019, 83, No. e00074-18.

(5) Nauhaus, K.; Boetius, A.; Kruger, M.; Widdel, F. In vitro demonstration of anaerobic oxidation of methane coupled to sulphate reduction in sediment from a marine gas hydrate area. Environ. Microbiol. 2002, 4, 296-305.

(6) Nauhaus, K.; Treude, T.; Boetius, A.; Kruger, M. Environmental regulation of the anaerobic oxidation of methane: a comparison of ANME-I and ANME-II communities. Environ. Microbiol. 2005, 7, 98-106.

(7) Timmers, P. H. A.; Welte, C. U.; Koehorst, J. J.; Plugge, C. M.; Jetten, M. S.; Stams, A. J. Reverse Methanogenesis and Respiration in Methanotrophic Archaea. Archaea 2017, 2017, 1654237.

(8) Knittel, K.; Losekann, T.; Boetius, A.; Kort, R.; Amann, R. Diversity and distribution of methanotrophic archaea at cold seeps. Appl. Environ. Microbiol. 2005, 71, 467-479.

(9) Meyerdierks, A.; Kube, M.; Kostadinov, I.; Teeling, H.; Glöckner, F. O.; Reinhardt, R.; Amann, R. Metagenome and mRNA expression analyses of anaerobic methanotrophic archaea of the ANME-1 group. Environ. Microbiol. 2010, 12, 422-439.

(10) Krukenberg, V.; Riedel, D.; Gruber-Vodicka, H. R.; Buttigieg, P. L.; Tegetmeyer, H. E.; Boetius, A.; Wegener, G. Gene expression and ultrastructure of meso- and thermophilic methanotrophic consortia. Environ. Microbiol. 2018, 20, 1651-1666.

(11) Krukenberg, V.; Harding, K.; Richter, M.; Glöckner, F. O.; Gruber-Vodicka, H. R.; Adam, B.; Berg, J. S.; Knittel, K.; Tegetmeyer, H. E.; Boetius, A.; Wegener, G. Candidatus Desulfofervidus auxilii, a hydrogenotrophic sulfate-reducing bacterium involved in the thermophilic anaerobic oxidation of methane. Environ. Microbiol. 2016, 18, 3073-3091.

(12) Wegener, G.; Krukenberg, V.; Ruff, S. E.; Kellermann, M. Y.; Knittel, K. Metabolic Capabilities of Microorganisms Involved in and Associated with the Anaerobic Oxidation of Methane. Front. Microbiol. 2016, 7, 46.

(13) Wegener, G.; Krukenberg, V.; Riedel, D.; Tegetmeyer, H. E.; Boetius, A. Intercellular wiring enables electron transfer between methanotrophic archaea and bacteria. Nature 2015, 526, 587-590.

(14) Scheller, S.; Yu, H.; Chadwick, G. L.; McGlynn, S. E.; Orphan, V. J. Artificial electron acceptors decouple archaeal methane oxidation from sulfate reduction. Science 2016, 351, 703-707.

(15) Skennerton, C. T.; Chourey, K.; Iyer, R.; Hettich, R. L.; Tyson, G. W.; Orphan, V. J. Methane-Fueled Syntrophy through Extracellular Electron Transfer: Uncovering the Genomic Traits Conserved within Diverse Bacterial Partners of Anaerobic Methanotrophic Archaea. mBio 2017, 8, No. e00530-17.

(16) McGlynn, S. E.; Chadwick, G. L.; Kempes, C. P.; Orphan, V. J. Single cell activity reveals direct electron transfer in methanotrophic consortia. Nature 2015, 526, 531-535.

(17) Holler, T.; Widdel, F.; Knittel, K.; Amann, R.; Kellermann, M. Y.; Hinrichs, K.-U.; Teske, A.; Boetius, A.; Wegener, G. Thermophilic anaerobic oxidation of methane by marine microbial consortia. ISME J. 2011, 5, 1946-1956.

(18) Abken, H.-J.; Tietze, M.; Brodersen, J.; Bäumer, S.; Beifuss, U.; Deppenmeier, U. Isolation and characterization of methanophenazine and function of phenazines in membrane-bound electron transport of Methanosarcina mazei Go1. J. Bacteriol. 1998, 180, 2027-2032.

(19) Jiang, X.; Ling, X.; Han, F.; Li, R.; Cui, J. Analysis of 4-methylpiperazine-1-carbodithioic acid 3-cyano-3,3-diphenyl-propyl ester hydrochloride and its major metabolites in rat plasma and tissues by LC-MS/MS. J. Sep. Sci. 2007, 30, 2080-2085.

(20) Weidolf, L. O. G.; Lee, E. D.; Henion, J. D. Determination of boldenone sulfoconjugate and related steroid sulfates in equine urine by high-performance liquid chromatography/tandem mass spectrometry. Biomed. Environ. Mass Spectrom. 1988, 15, 283-289. 
(21) de Loor, H.; Poesen, R.; De Leger, W.; Dehaen, W.; Augustijns, P.; Evenepoel, P.; Meijers, B. A liquid chromatography - tandem mass spectrometry method to measure a selected panel of uremic retention solutes derived from endogenous and colonic microbial metabolism. Anal. Chim. Acta 2016, 936, 149-156.

(22) Oppernheimer, N. J. Chemistry and Solution Conformation of the Pyridine coenzymes. In The Pyridine Nucleotide Coenzymes; Everse, J., Ed.; Academic Press: New York, 1992; pp 51-89.

(23) Curphey, T. J.; Prasad, K. S. Diquaternary salts. I. Preparation and characterization of the diquaternary salts of some diazines and diazoles. J. Org. Chem. 1972, 37, 2259-2266.

(24) Diculescu, V. C.; Militaru, A.; Shah, A.; Qureshi, R.; Tugulea, L.; Brett, A. M. O. Redox mechanism of lumazine at a glassy carbon electrode. J. Electroanal. Chem. 2010, 647, 1-7.

(25) Han, J.; Yen, S.; Han, G.; Han, P. Quantitation of hydrogen peroxide using tris(2-carboxyethyl)phosphine. Anal. Biochem. 1996, 234, 107-109.

(26) Morton, R. A. Biochemistry of Quinones; Academic Press: London, New York, 1965; p 585.

(27) Jagendorf, A.; Margulies, M. Inhibition of spinach chloroplast photosynthetic reactions by p-chlorophenyl-1,1-dimethylurea. Arch. Biochem. Biophys. 1960, 90, 184-195.

(28) Friedheim, E.; Michaelis, L. Potentiometric study of pyocyanine. J. Biol. Chem. 1931, 91, 355-368.

(29) Bahner, C. T.; Norton, L. L. Some Quaternary Salts of Pyrazine. J. Am. Chem. Soc. 1950, 72, 2881-2882.

(30) Easley, W. K.; Bahner, C. T. Some Quaternary Ammonium Salts of Quinoxalines. J. Am. Chem. Soc. 1950, 72, 3803.

(31) Kaim, W. Flavosemiquinone model systems. Part 1. 4-Hydro-1alkylpyrazinium radical cations. J. Chem. Soc., Perkin Trans. 2 1984, 1357-1359.

(32) Kaim, W. Flavosemiquinone model systems. Part 2. Methylsubstituted quinoxaline radical ions. J. Chem. Soc., Perkin Trans. 2 1984, 1767-1769.

(33) Green, D. E.; Mii, S.; Kohout, P. M. Studies on the terminal electron transport system. I. Succinic dehydrogenase. J. Biol. Chem. 1955, 217, 551-567.

(34) Wheaton, G. A.; Stoel, L. J.; Stevens, N. B.; Frank, C. W. Optical Spectra of Phenazine, 5,10-Dihydrophenazine, and the Phenazhydrins. Appl. Spectrosc. 1970, 24, 339-343.

(35) Wang, Y.; Newman, D. K. Redox reactions of phenazine antibiotics with ferric (hydr)oxides and molecular oxygen. Environ. Sci. Technol. 2008, 42, 2380-2386.

(36) Klatt, L. N.; Rouseff, R. L. Electrochemical Reduction of Pyrazine in Aqueous Media. J. Am. Chem. Soc. 1972, 94, 7295-7304.

(37) Armand, J.; Chekir, K.; Pinson, J. Titanium(III) Chloride and Electrochemical Reduction of Pyrazine, Quinoxaline and Triazine Derivatives and of Their Salts. J. Heterocycl. Chem. 1980, 17, 12371240.

(38) Bailey, D. N.; Hercules, D. M.; Roe, D. K. Electrochemistry and Photopotentials of Phenazine in Methanol Solutions. J. Electrochem. Soc. 1969, 116, 190-195.

(39) Lunn, G. Reduction of heterocycles with nickel-aluminum alloy.

J. Org. Chem. 1987, 52, 1043-1046.

(40) Hamer, J.; Holliday, R. E. On the Reduction of Quinoxaline. J. Org. Chem. 1963, 28, 2488.

(41) Armand, J.; Chekir, K.; Pinson, J. Electrochemical Reduction of Pyrazines into Dihydropyrazines. Preparation of Dihydropyrazines. Can. J. Chem. 1974, 52, 3971-3980.

(42) Rupar, J.; Aleksić, M. M.; Nikolić, K.; Popović-Nikolić, M. R. Comparative electrochemical studies of kinetic and thermodynamic parameters of Quinoxaline and Brimonidine redox process. Electrochim. Acta 2018, 271, 220-231.

(43) Moore, J. A.; Capaldi, E. C. The Oxidative Cyclization of 2,5Dihydroxyphenylalkylamines to 5-Hydroxyindoles and 6-Hydroxyquinolines1. J. Org. Chem. 1964, 29, 2860-2864.

(44) Chen, Q.-A.; Wang, D.-S.; Zhou, Y.-G.; Duan, Y.; Fan, H.-J.; Yang, Y.; Zhang, Z. Convergent Asymmetric Disproportionation
Reactions: Metal/Brønsted Acid Relay Catalysis for Enantioselective Reduction of Quinoxalines. J. Am. Chem. Soc. 2011, 133, 6126-6129.

(45) Takano, Y.; Kaneko, M.; Kahnt, J.; Imachi, H.; Shima, S.; Ohkouchi, N. Detection of coenzyme F430 in deep sea sediments: A key molecule for biological methanogenesis. Org. Geochem. 2013, 58, 137-140.

(46) Deng, X.; Dohmae, N.; Nealson, K. H.; Hashimoto, K.; Okamoto, A. Multi-heme cytochromes provide a pathway for survival in energy-limited environments. Sci. Adv. 2018, 4, No. eaao5682.

(47) Thauer, R. K.; Jungermann, K.; Decker, K. Energy conservation in chemotrophic anaerobic bacteria. Bacteriol. Rev. 1977, 41, 100180.

(48) Bond, D. R.; Lovley, D. R. Reduction of Fe(III) oxide by methanogens in the presence and absence of extracellular quinones. Environ. Microbiol. 2002, 4, 115-124.

(49) Yan, Z.; Joshi, P.; Gorski, C. A.; Ferry, J. G. A biochemical framework for anaerobic oxidation of methane driven by $\mathrm{Fe}(\mathrm{III})$ dependent respiration. Nat. Commun. 2018, 9, 1642.

(50) Straub, K.; Benz, M.; Schink, B. Iron metabolism in anoxic environments at near neutral pH. FEMS Microbiol. Ecol. 2001, 34, 181-186.

(51) Prast-Nielsen, S.; Huang, H.-H.; Williams, D. L. Thioredoxin glutathione reductase: its role in redox biology and potential as a target for drugs against neglected diseases. Biochim. Biophys. Acta 2011, 1810, 1262-1271.

(52) Widdel, F. B.; Bak, F. Gram-negative mesophilic sulfatereducing bacteria. In The Prokaryotes, 2nd ed.; Balows, A., Trüper, H. G., Dworkin, M., Harder, W., Schleifer, K.-H., Eds.; Springer: New York, 2002.

(53) Laso-Pérez, R.; Krukenberg, V.; Musat, F.; Wegener, G. Establishing anaerobic hydrocarbon-degrading enrichment cultures of microorganisms under strictly anoxic conditions. Nat. Protoc. 2018, 13, 1310-1330.

(54) Fahey, R. C.; Newton, G. L. Determination of low-molecularweight thiols using monobromobimane fluorescent labeling and highperformance liquid chromatography. Methods Enzymol. 1987, 143, $85-96$.

(55) Rethmeier, J.; Rabenstein, A.; Langer, M.; Fischer, U. Detection of traces of oxidized and reduced sulfur compounds in small samples by combination of different high-performance liquid chromatography methods. J. Chromatogr. A 1997, 760, 295-302.

(56) Allen, K. D.; White, R. H. Occurrence and biosynthesis of 3mercaptopropionic acid in Methanocaldococcus jannaschii. FEMS Microbiol. Lett. 2016, 363, fnw217.

(57) Yang, C.; Jin, Q.; Zhang, H.; Liao, J.; Zhu, J.; Yu, B.; Deng, J. Tetra-(tetraalkylammonium)octamolybdate catalysts for selective oxidation of sulfides to sulfoxides with hydrogen peroxide. Green Chem. 2009, 11, 1401-1405.

(58) Li, J.-T.; Ao, Y.-F.; Wang, Q.-Q.; Wang, D.-X.; Wang, M.-X. Synthesis and structure of $\mathrm{N}$-methylated azacalix[4]pyridines and azacalix[1] arene[3]pyridines. Tetrahedron Lett. 2017, 58, 3708-3711. 